19 Revue d'histoire du XIXe siècle

Société d'histoire de la révolution de 1848 et des

révolutions du XIXe siècle

$40 \mid 2010$

Discours

\title{
Politique et économie politique chez Pierre et Jules Leroux
}

Pierre and Jules Leroux's politics and political economics

Politik und politische Wirtschaft bei Pierre und Jules Leroux

Ludovic Frobert

\section{CpenEdition}

Journals

Édition électronique

URL : http://journals.openedition.org/rh19/3992

DOI : 10.4000/rh19.3992

ISSN : $1777-5329$

Éditeur

La Société de 1848

Édition imprimée

Date de publication : 15 juillet 2010

Pagination : 77-94

ISSN : 1265-1354

Référence électronique

Ludovic Frobert, "Politique et économie politique chez Pierre et Jules Leroux », Revue d'histoire du XIXe siècle [En ligne], 40 | 2010, mis en ligne le 15 juillet 2013, consulté le 01 mai 2019. URL : http:// journals.openedition.org/rh19/3992 ; DOI : 10.4000/rh19.3992 


\section{LUDOVIC FROBERT}

\section{Politique et économie politique chez Pierre et Jules Leroux}

On considère encore aujourd'hui que l'article "économie politique" signé par Pierre Leroux (1797-1871) et qui paraît lors de l'été 1834 dans la Revue encyclopédique contient l'une des toutes premières occurrences en français du terme "socialisme». Le socialisme est alors opposé à l'individualisme et Leroux renvoie dos-à-dos les deux systèmes. Le "Socialisme», explique-t-il, est un «système exclusif», qui sacrifie la liberté à l'organisation; il est le symétrique de «l'Individualisme», système où tout gouvernement et tout contrôle sont sacrifiés à une prétendue liberté sans borne : «Tandis que les partisans de l'individualisme se réjouissent ou se consolent sur les ruines de la société, réfugiés qu'ils sont dans leur égoïsme, les partisans du socialisme, marchant bravement à ce qu'ils nomment une époque organique, s'évertuent à trouver comment ils enterreront toute liberté, toute spontanéité, sous ce qu'ils nomment l'organisation " ${ }^{1}$. Dans les faits, les deux systèmes pourraient d'ailleurs ne pas différer grandement, Leroux signalant qu'au titre du socialisme la secte saint-simonienne a autorisé des libertés souvent curieuses voire scabreuses pour ses principales "capacités ", et que l'individualisme, que célèbrent Doctrinaires et autres éclectiques vendus désormais au régime de Juillet, se traduit au présent, par un "despotisme pygmée » ${ }^{2}$ à ces deux systèmes Leroux oppose donc celui de l'Association ${ }^{3}$, un système garantissant la solidarité, car conciliant sans relâche liberté et égalité et se situant dès lors dans le sens du progrès continu de l'humanité.

L'article "économie politique» dans lequel Leroux écrit notamment que "c'est sa dignité, c'est sa qualité d'homme, c'est sa liberté, c'est son indépendance, que le prolétaire revendique lorsqu'il aspire à posséder des biens matériels" permet en partie de nuancer l'image d'un penseur de (trop) haut

1. Pierre Leroux, «Economie politique. Cours d'économie politique fait à l'Athénée de Marseille par M. Jules Leroux", Revue encyclopédique, octobre-décembre 1833 (le volume paru durant l'été 1834), p. 107-108.

2. Pierre Leroux, «De la tendance nouvelle des idées», Revue encyclopédique, janvier 1832, p. 8.

3. Lopposition entre «Individualisme» et «Association» est proposée par Leroux dans «De la philosophie et du christianisme", Revue encyclopédique, août 1832, p. 281-340 (reproduit dans Pierre Leroux, Euvres, tome premier, Paris, Société typographique, 1850). 
vol, d'un philosophe mystique. Mais d'autres précisions et rectifications s'imposent peut-être à propos de ce texte clé publié en 1834. Il est, par exemple, important de bien définir son statut; de remarquer que cette vingtaine de pages préfacent un autre texte. Pierre Leroux est coutumier de cette pratique dans les pages de sa revue, une revue qui se veut encyclopédique et qui vise donc à unifier, circonscrire toutes les connaissances autour d'une doctrine, la sienne. Ces présentations insèrent donc ces documents au sein de la doctrine du progrès continu, interprétant et traduisant ces fragments, les critiquant parfois. Une réelle distance, reconnue ou non, n'est donc pas impossible entre la présentation et le fragment lui-même. Les choses sont plus compliquées encore ici car la présentation de Leroux précède un texte de son jeune frère Jules Leroux (1805-1883). Très peu d'études ont été consacrées à ce personnage ${ }^{4}$. On sait qu'il exerçait, plus assidûment encore que Pierre, la profession d'imprimeur, qu'il suivit l'aventure saint-simonienne et celle de l'Encyclopédie nouvelle, et qu'il participa avec la fratrie des Leroux au phalanstère de Boussac et au succès de la Revue indépendante puis de la Revue sociale dans la première partie des années 1840. En 1848 Jules Leroux fut député de la Creuse à l'Assemblée législative, radical, siégeant sur les bancs de la Montagne et se faisant surnommer le "petit Robespierre». En 1851, il suivit encore Pierre en exil à Londres puis à Jersey avant d'émigrer avec toute sa famille, une quinzaine d'années plus tard aux États-Unis, rejoignant les communautés icariennes au Kansas, en Iowa puis en Californie; c'est là qu'il renoua encore avec sa mission d'imprimeur, de journaliste et de rédacteur, faisant vivre plusieurs journaux radicaux, organes, selon ses dires, de la vraie république française en exil, de L'Étoile du Kansas (1873-1880) à L'Étoile des pauvres et des souffrants (1881-1883). Des quelques rapides mentions ${ }^{5}$ que l'on trouve aujourd'hui de l'action et de l'œuvre de Jules Leroux ressortent deux impressions, pas forcément entièrement juxtaposables : la première est celle d'un simple lieutenant de son prestigieux aîné. On évoque une division du travail, Pierre étant le grand coordinateur, Jules le responsable du polissage de la seule partie «économique» de la doctrine. Mais on mentionne aussi à propos du cadet une trajectoire singulière, plus laborieuse, plus tumultueuse, et on se réfere encore le plus souvent aux mots d'Henri Mougin repérant chez Jules une «inspiration rude, et plus prolétarienne».

4. On ne dispose vraiment que de : Nadine Dormoy Savage, "Jules Leroux en Icarie», The French Review, vol. 49, n6, mai 1976, p. 1025-1040.

5. Mentions notamment chez Leonardo La Puma, Il socialismo sconfito, Milano, Franco Angeli, 1984, p. 118; Marisa Forcina, I diritti dell'esistente. La filosofia della "Encyclopédie nouvelle" 18331847, Lecce, Milella, 1987, p. 10 et 24; Jean-Pierre Lacassagne, Histoire d'une amitié. Pierre Leroux et Georges Sand, Paris, Klincksieck, 1973, p. 84-87; Pierre Leroux, À la source perdue du socialisme français, anthologie établie et présentée par Bruno Viard, Paris, Desclée de Brower, 1997; Vincent Peillon, Pierre Leroux et le socialisme républicain, Latresne, Le Bord de l'Eau, 2003. Ces auteurs se réferent le plus souvent au passage qu'Henri Mougin consacre à Jules Leroux dans son ouvrage de 1938 (Henri Mougin, Pierre Leroux, Paris, Éditions sociales internationales, 1938, p. 117-129); Mougin présentait une analyse d'articles économiques publiés par Jules Leroux dans la Revue sociale en 1850. 
Un écart, quelques frottements sont donc possibles entre la doctrine générale de Pierre, une religion de l'Humanité, et la réflexion économique de Jules. Et il n'est pas impossible qu'un véritable travail, et pas simplement une division hiérarchique du travail, ait eu lieu là, entre les deux frères, au croisement du politique et de l'économique ${ }^{6}$. Nous étudierons ce travail de frottement en nous limitant à la période 1831-1835, période de bouleversements politiques, économiques, communicationnels mais aussi période de première maturité de la pensée des deux frères. Nous proposerons une lecture attentive d'un corpus limité de textes en nous centrant sur l'articulation économie/politique. Pierre et Jules Leroux s'accordent alors sur le fait que l'émancipation économique et sociale doit être l'une des toutes premières mesures du progrès général et doit donc figurer à rang égal avec la conquête de certaines libertés civiles et politiques. Mais, s'il y a accord sur le but entre Pierre et Jules Leroux, ils se séparent sur les moyens pour réaliser et pérenniser cette émancipation : Pour Pierre, la liberté économique procédera chronologiquement et logiquement des avancées de la liberté politique : c’est la constitution d'un État central démocratique qui assurera le progrès économique; pour Jules, qui ne nie pas l'apport que pourra constituer un gouvernement républicain, ce sont aussi, et surtout, des transactions économiques elles-mêmes, et notamment de la croissance des associations, coopératives, mutuelles ouvrières que procéderont émancipation économique, sociale et finalement politique.

\section{Politique et économie chez Pierre Leroux}

Dans la seconde partie de l'année 1831, rompant désormais avec les saintsimoniens après s'être déjà éloigné des Doctrinaires libéraux, Pierre Leroux rejoint l'équipe de la Revue encyclopédiquę. Les commentateurs considèrent que les articles et notes qu'il publie les quatre années suivantes dans cette revue composent un premier état stable, complet et cohérent de sa doctrine du progrès continu. Il n'est pas question ici de revenir sur l'œuvre synthétique de Leroux, plusieurs études classiques faisant référence ${ }^{8}$. Nous limiterons donc ici notre enquête à l'articulation économie-politique chez Leroux.

Au lendemain désenchanté de Juillet, Leroux observe une «société silencieuse», "en poussière " ${ }^{9}$, sans croyances communes pouvant motiver et

6. Voir ici l'importante lettre critique adressée à Jules Leroux par Jean Reynaud vers 1835 (Jean Reynaud, Correspondance familière, Paris, 1866, p. 117-119).

7. Jean-Jacques Goblot, Aux origines du socialisme français : Pierre Leroux et ses premiers écrits, Lyon, Presses universitaires de Lyon, 1977.

8. Il faut retenir surtout Miguel Abensour, "Pierre Leroux et l'utopie socialiste», Économie et sociétés, décembre 1972, p. 2201-2247; Armelle Le Bras-Chopard, De l'égalité dans la différence. Le socialisme de Pierre Leroux, Paris, Presses de la FNSP, 1986.

9. Pierre Leroux, "Religion. Aux philosophes», Revue encyclopédique, septembre 1831, p. 499. 
orienter l'action collective; une société à qui, niant ce qui est en germe dans l'humanité - une capacité évoluée d'adaptation à son milieu allant jusqu'à sa réforme -, on a encore menti sur son aptitude à mener à bien des projets et à délibérer sur des choix collectifs. Au règne négocié de la fraternité, qui cultive le dialogue entre liberté et égalité, on a opposé et imposé un évolutionnisme aveugle, chaos et hasard réglant les trajectoires économiques et politiques des individus pour le plus grand bien de tous. Il faut alors selon Leroux récuser ce providentialisme et reformuler un projet de développement grâce à une nouvelle "pensée organique et constitutive de la société». Cette nouvelle synthèse fruit d'un encyclopédisme fondé sur "la liberté, l'égalité et la science» tirant ses enseignements de trois siècles d'émancipation scientifique, littéraire et religieuse - mais dont le XVIII siècle fut le "point fixe " converge donc vers l'idée de fraternité, considérée ici comme une virtualité, propre à l'homme, à définir et mener des actions communes. Leroux l'écrit d'ailleurs magnifiquement, cernant ici l'objet de la philosophie : «Le problème de la philosophie n'est pas de constituer la raison individuelle de chaque homme, indépendamment de toute condition de temps et d'époque, et dans une ère d'absolue; mais de constituer et d'organiser la raison collective de l'humanité vivante $»^{10}$. Cultiver cette raison collective, c'est la faire vivre, la vie n'étant pas simple "accumulation", mais "création incessante ${ }^{11}$. Leroux tire ici profit d'un modèle transformiste qui, de Lamarck à Geoffroy Saint-Hilaire lui a enseigné que la vie était un effet de l'organisation et que son développement dépendait du traitement par chaque vivant des instructions du milieu ${ }^{12}$. Le développement de l'humanité nécessite donc une multiplication des agrégations et relations pour former le milieu social et pour exploiter le milieu naturel (temps et espace), une condition que ne peut naturellement pas remplir un état social présent simple «amas d'égoïsmes» et dans lequel les $9 / 10^{e}$ de la population vit dans la précarité et la misère; dans lequel donc, en quantité et en qualité, les relations sociales sont quasiment inexistantes. "C'est la société, c'est l'union des hommes entre eux, c'est l'organisation qui produit la richesse» rappelle Leroux qui dès lors peut définir le but du politique par "l'avènement et l'élévation du prolétariat", du plus grand nombre; c'est en élevant le plus grand nombre qu'on a, en effet, logiquement, le plus de chance de multiplier et de varier les relations. Revendiquant ici l'héritage de Saint-Simon, il note encore, «il n'y a de révolutions durables et fécondes, de révolutions accomplies sans retour, que celles qui intéressent directement

10. Pierre Leroux, «De la loi de continuité qui unit le $18^{\mathrm{e}}$ siècle au $17^{\mathrm{e}}$ siècle», Revue encyclopédique, mars 1833, p. 497.

11. Pierre Leroux, "Des rapports du christianisme avec la doctrine philosophique du progrès", Revue encyclopédique, janvier 1835 , p. 84.

12. Sur ce point, voir notamment Giulio Barsanti, «Lamarck et la naissance de la biologie», in Goulven Laurent [dir.], Lamarck (1744-1829), Paris, Éditions du CTHS, 1997; Pietro Corsi, Lamarck. Genèse et enjeux du transformisme, 1770-1830, Paris, CNRS Éditions, 2001. 
la classe nombreuse et laborieuse, qui améliorent radicalement sa condition morale et matérielle ${ }^{13}$.

La définition d'une société fraternelle, composée d'hommes libres et égaux transformant en commun leur milieu, a été partiellement perçue et dégagée depuis le $\mathrm{XVI}^{e}$ siècle par des générations de savants et de philosophes, et nombre de moyens de réalisation ont été proposés. Leroux montre qu'une succession d'efforts partiels a nourri une véritable "tradition" de l'émancipation qui permet de relier passé, présent et avenir. L'Individualisme fut l'un de ces efforts. Leroux suggère que l'Individualisme a ainsi longtemps servi à bousculer une conception autocratique du pouvoir et à déstabiliser un monde où dominait «l'association théologique-féodale ${ }^{14}$. Cet individualisme trouve, dans le présent, en France, deux prolongements : en philosophie avec l'éclectisme de Théodore Jouffroy et en économie politique avec l'industrialisme de Jean-Baptiste Say. La dégénérescence de ces deux programmes, vulnérables l'un et l'autre à la corruption de Juillet, permet de signaler l'erreur qui dès l'origine était au cœur de l'Individualisme et explique que dans le présent il a épuisé ses effets positifs. Avec Descartes, en effet, c'est la raison individuelle qui doit être le moteur de l'émancipation et Descartes, selon Leroux, fait accomplir un grand pas en avant à la tradition de l'émancipation. Toutefois cette raison, remarque Leroux, demeure ici une raison isolée, sans boussole car sans relation aux milieux et aux obstacles : une raison qui se développerait sans relation aux autres, et sans relation au milieu naturel, temps et espace. Le doute cartésien n'est donc pas réel et cette raison est incapable de se confronter aux réalités, incapable de définir but et moyens dans des situations de doute réel. La philosophie, rappelle Leroux (s'appuyant ici encore sur Geoffroy Saint-Hilaire) est «la science du tout, la science de la vie» et il lui faut donc introduire «l'élément du temps, et avec lui la progression, la suite, la conséquence $»^{15}$. Le temps, l'histoire enseignent ici ce que Leroux appelle des "expériences» et des "consentements», les premières relevant des rapports homme-milieu naturel, les secondes du rapport homme-milieu social. C'est la somme de ces expériences et surtout de ces consentements (que Leroux associe à la capacité cumulée de jugement dans les affaires humaines, dans les situations de coopération/conflits), et l'enseignement général qui s'en dégage, qui fournissent sens et repères aux actions présentes et aux aspirations à venir : «le principe général de certitude dans l'ordre de la vie humaine collective est le consentement actuel, manifesté par la tradition actuelle de l'humanité ${ }^{16}$. On comprend alors mieux les raisons de la faillite de l'économie politique smithienne dans la version qu'en propose

13. Pierre Leroux, «De la tendance nouvelle des idées», loc. cit., p. 2-3.

14. Pierre Leroux, "Des rapports du christianisme avec la doctrine philosophique du progrès ", loc. cit., p. 305.

15. Pierre Leroux, "Préface. Aux souscripteurs de la revue», Revue encyclopédique, octobre-décembre 1833, p. XX -XXI.

16. Idem, p. LVIII. 
Say. L'industrialisme libéral, traduction économique de l'Individualisme, est un régime proprement "impuissant ${ }^{17}$; Leroux évoquant ici une économie "libérale en apparence, meurtrière en réalité ${ }^{18}$. Coupée de la vie, armée de la seule certitude de l'individu-propriétaire et de sa rationalité s'exprimant dans les situations actuelles de concurrence, cette économie n'a aucun moyen de juger le présent et ne peut proposer qu'une pseudo-observation. L'économie politique de Say, n'est en effet, selon Leroux, «autre chose que la constatation des phénomènes bien ou mal entrevus qui résultaient fatalement d'une agglomération d'hommes fondée sur un principe directement contraire à l'idée de société, si on peut appeler un principe l'isolement, la lutte, l'individualisme $»^{19}$. Impuissante à juger, cette économie devient plus ou moins volontairement complice du pouvoir et de l'oppression, reproduisant sous une forme nouvelle, le prolétariat, «l'exploitation de l'homme par l'homme ${ }^{20}$. Ce qu'ajournent donc Individualisme et Libéralisme, c'est une véritable réflexion en économie, en morale et en politique, sur le gouvernement de l'Humanité. En économie, les premiers à récemment combler ce manque ont été les saintsimoniens, ceux que, dans son texte de 1834, Leroux identifie au Socialisme. Les socialistes s'engagent dans le dernier combat en faveur de l'émancipation, résumé par les mots de Saint-Simon : «Toutes les institutions sociales doivent avoir pour but l'amélioration morale, intellectuelle et physique, de la classe la plus nombreuse et la plus pauvre». Les saint-simoniens comprennent que, sous couvert d'émancipation, l'Individualisme livre «les classes inférieures à la plus brutale exploitation $»^{21}$ et ils font dès lors de la résolution de ce problème une question de gouvernement, ou, dans leurs propres termes, d'administration et d'aménagement. Renouant, sans l'avouer, avec le XVIII siècle et la Révolution, ils localisent la solution au niveau d'un contrôle collectif de la propriété, considérée comme une "création sociale»"22. La contribution socialiste à la doctrine du progrès est donc double et réside dans la mise au premier rang de la question sociale assimilée à un problème de contrôle de la propriété et dans le choix d'en faire un problème de gouvernement. Pourtant, selon Leroux, le programme socialiste, comporte une erreur. L'Individualisme avait le défaut de miser exclusivement sur le présent et sur la raison individuelle. Le Socialisme a lui aussi une vision tronquée du progrès continu, de la vie, qui, symétriquement, nie le présent, pour ne solliciter qu'un curieux mélange de passé et d'avenir; dès lors les incessants bricolages et autres praxis quotidiennes sont évacués de ce programme. Corrélativement, le Socialisme

17. «Plus de libéralisme impuissant», titre d'un article qu’avait publié Pierre Leroux dans Le Globe en janvier 1831 .

18. Pierre Leroux, «De la philosophie et du christianisme», loc. cit., p. 303.

19. Idem, p. 303-304.

20. Idem, p. 306.

21. Ibidem.

22. Doctrine de Saint-Simon. Exposition, Paris, au Bureau de l'Organisateur, 1830 (Première année 1829; deuxième année 1829-1830). Pour la critique de la propriété, voir surtout les chapitres 6-8 de la première année. 
promeut une sorte de raison collective qui ne procède pas de l'expérience cumulée d'échanges, discussions ou communications horizontales entre individus, mais d'une révélation verticale que des «capacités» scientifiques ou industrielles transmettent aux masses. En résumé, les socialistes ont eu le tort d'ignorer institutions et valeurs de liberté, « de ne pas voir leur immense utilité et leur absolue nécessité pour faire triompher les intérêts de la classe la plus nombreuse et la plus pauvre, en transformant les idées par la discussion, et en permettant au génie de l'innovation de présenter, dans la triple direction de la politique, de la science et de l'art, toutes les inspirations qui peuvent servir de véhicule et d'aliments à cette transformation »23. Mais Leroux exprime aussi beaucoup brutalement sa répugnance à l'égard de cette nouvelle papauté, écrivant, par exemple, qu'Enfantin, le "père», "a produit une sorte de procédé mécanique pour construire, à volonté, de force et par une vraie fantasmagorie, des hommes nouveaux, une société nouvelle ${ }^{24}$.

Le Socialisme, qui a le grand mérite de promouvoir la question sociale et d'en faire une question de direction collective de la société, ne comprend donc pas le vrai sens du mot gouverner. De leur côté les républicains, en plein tumulte lors des premiers trimestres succédant à juillet 1830, tâtonnent autour d'une bonne définition du gouvernement. Mais oubliant les enseignements de 1793, ils ne voient encore dans la question sociale "qu'un combat là où il s'agit du problème de la législation tout entière ${ }^{25}$. Selon Leroux, seuls Jean Reynaud ou Pierre-Mathieu Laurent, ses collègues de la Revue encyclopédique, ont correctement posé et résolu le problème ${ }^{26}$. Deux grandes propositions, endossées par Leroux, sont avancées :

- le Socialisme n'étant qu'une dérive assez sectaire, le grand obstacle au présent demeure l'Individualisme, doctrine des intérêts bourgeois : «la classe bourgeoise, la classe propriétaire, la classe qui tient par intérêt et par sentiment au système d'individualisme pur, voilà donc l'ennemi! ${ }^{27}$. Une lutte est donc à prévoir contre cette classe et sa doctrine, et seule «la doctrine de l'Association, la doctrine de la révolution française, la doctrine de l'égalité organisée» peut lui être opposée. Si le but, l'Association, est nettement affirmé28, reste à déterminer les moyens. Or, selon Leroux, seule la législation, le "progrès législatif», peut permettre de saper l'Individualisme. Ce progrès passe d'abord, et avant tout, par une réforme du

23. Idem, p. 312.

24. Pierre Leroux, «Des rapports du christianisme avec la doctrine philosophique du progrès », loc. cit., p. 145.

25. Pierre Leroux, "De la philosophie et du christianisme», loc. cit, p. 312.

26. Jean Reynaud, "De la nécessité d'une représentation spéciale pour les prolétaires», Revue encyclopédique, avril 1832; Pierre-Mathieu Laurent, "Le tiers-état et les prolétaires ", Revue encyclopédique, octobre 1832.

27. Pierre Leroux, «De la philosophie et du christianisme», loc. cit., p. 317.

28. Pierre Leroux, "Du progrès législatif», loc. cit., p. 259. Il poursuit : «L'égalité dans et par l'association, voilà où nous conduisent toutes les avenues du passé, où tendent tous les efforts du présent, où mènent toutes les lueurs que nous jettent l'avenir». 
législateur; il faut ici récuser l'illusion des capacités, aussi bien censitaires qu'industrielles ou scientifiques, et mettre en place un «législateur multiple» : "pour nous le législateur est donc muable et progressif, comme la loi elle-même ${ }^{29}$. $\mathrm{La}$ «réforme parlementaire» constitue donc une priorité du "programme de la politique», logiquement et chronologiquement : "d'abord et avant tout le perfectionnement du gouvernement représentatif par la réforme parlementaire... cette question de la représentation véritable est à nos yeux la pierre fondamentale de l'édifice ${ }^{30}$.

- C'est cette forme gouvernementale qui va entraîner le fond de la «réforme sociale ${ }^{31}$. Selon Leroux, il est important de remarquer que même dans une conjoncture politique aussi peu démocratique et progressiste que celle du ministère du Comte d'Argout ${ }^{32}$, les quelques avancées de la représentativité permises par la Révolution de 1830 ont entraîné une vague de réformes lors des dernières sessions législatives : projets de canaux et chemins de fer, loi d'expropriation forcée pour faciliter ces travaux, fondation de colonies agricoles, etc. Tout progrès dans la représentation politique favorise les réformes et notamment celles conduisant à une remise en question du droit individuel absolu de propriété. Leroux décrit donc une transformation (le progrès législatif) dans laquelle aux progrès dans la forme du gouvernement (le législateur) répondent les avancées de la réforme (la loi). Évoquant cette dynamique entre forme de gouvernement et réforme, Leroux écrit : "réunies, elles se prêtent un mutuel appui, et constituent le progrès politique et social; séparées, elles se détruisent d'elles-mêmes, et perdent toute réalité. Considérer le gouvernement représentatif en lui-même et comme un but, au lieu de l'aimer comme un instrument de progrès, c'est le stériliser, c'est en faire un jouet inutile, c'est l'anéantir. S'occuper de réformes législatives, désirer des améliorations sociales, sans idée arrêtée sur le gouvernement politique, sur ses progrès, sans penser et sans croire que les idées jetées dans le sein du peuple feront naître et grandir des législateurs dignes de leur mission, c'est une absurde et creuse politique $»^{33}$. Leroux souligne finalement que, sur le terrain économique, cette progression liée du législateur et de la loi doit permettre de «socialiser les instruments de travail ${ }^{34}$.

Au milieu de l'année 1833, tiraillée par les dissensions entre ses Montagnards et ses Girondins, la Société des Droits de l'Homme demande à Pierre Leroux et Jean Reynaud de se charger de la rédaction de son manifeste. Leur Exposé des principes républicains de la Société des droits de l'homme et du

29. Idem, p. 260.

30. Idem, p. 262-263. Quelques lignes plus haut il écrit de même : «en théorie, comme en pratique, la question de la représentation du souverain, c'est-à-dire du peuple, est donc la clé de tout l'édifice social; c'est d'elle que jaillit toute force et toute lumière».

31. Idem, p. 267.

32. Le Comte d'Argout avait le portefeuille du Commerce et des Travaux publics dans le premier gouvernement du maréchal Soult.

33. Idem, p. 261.

34. Idem, p. 271. 
citoyen, synthétique, conciliant, n'en porte pas moins la trace de la doctrine qu'ils élaborent alors dans les pages de leur Revue encyclopédique : "Il faut que le peuple recouvre l'exercice de sa souveraineté» et il faut pour cela des principes politiques et une forme de gouvernement admettant «la discussion et le mouvement ${ }^{35}$. Sur le chapitre des principes, on ne peut que prendre exemple sur Robespierre et la Convention de 1793 et tenter de prolonger, de terminer, leurs efforts : "ce qui domine, résument Leroux et Reynaud, c'est la réparation des iniquités sociales, l'abolition des intérêts exclusifs et des usurpations qui les maintiennent, la garantie du droit de tous placé dans la souveraineté de tous, sans que l'individu perde son recours contre l'oppression; c'est en un mot le principe d'association appliqué à la société politique, c'est-à-dire l'égalité entre chacun des associés reconnus, protégée par le concours, et soumise uniquement aux conditions essentielles de la vie sociale ${ }^{36}$. Cette aspiration, cette vie, exprimées dans les principes de 1793 ne pouvaient être longtemps réprimées et, même dans un présent escamoté par le parti de l'Ordre, nombre de réformes sont en germe, réformes qui pourraient, sous un régime républicain, connaître une pleine extension : pouvoir central électif temporaire, assemblée nationale représentative des intérêts légitimes, organisation municipale, garde nationale, système d'éducation publique, organisation judiciaire, jury... Sur le terrain économique et social c'est un même progrès continu impulsé d'abord (là encore chronologiquement et logiquement) par un État devenu démocratique qui pourrait être responsable de l'émancipation : il faudrait, expliquent Leroux et Reynaud, pleinement développer un système financier faisant de l'État un acteur économique central, détenteur d'un important "capital social ${ }^{37}$; il faudrait également une véritable direction d'ensemble de la vie économique, les deux auteurs prévoyant ici "l'établissement de fonctions industrielles qui contribuent à réaliser ces deux grands principes, la meilleure division du travail, la meilleure répartition des produits, qui accélèrent l'émancipation de la classe ouvrière, et fassent intervenir la puissance et l'intelligence sociale dans le développement des intérêts sociaux ${ }^{38}$. Naturellement, Leroux et Reynaud, songeant ici à la multiplication en cours des différentes sociétés ouvrières - mutuelles et coopératives... - évoquent encore le "concours du droit d'association" aux progrès général de l'émancipation : mais, dans leur credo, l'Association renvoie d'abord et avant tout à la constitution d'un gouvernement central

35. Pierre Leroux et Jean Reynaud, Exposé des principes républicain de la Société des droits de l'homme et du citoyen, Paris, Herhnan, 1833, p. 1.

36. Idem, p. 4.

37. Ils évoquent ici "un système financier qui, ne se bornant pas à dégrever le pauvre, le travail, l'industrie, soit pourvu de ressources nécessaires pour les aider, les commanditer; qui remplace ce qu'on est convenu d'appeler les revenus de l'État par un capital social, dont la puissance constitue le budget public, non plus débiteur, mais créancier, et mette le crédit, non plus à la disposition des particuliers envers le corps social, mais à la disposition de celui-ci envers les particuliers», Idem, p. 5.

38. Idem, p. 6. 
démocratique, et l'émancipation économique et sociale dépend, là encore en priorité, des impulsions de ce pouvoir politique.

\section{L'économie politique de Jules Leroux}

Auteur de trois textes économiques dans la Revue encyclopédique en 18331834, Jules Leroux va immédiatement scander que "la question du siècle est une question sociale», qu'il faut se mobiliser "autour des questions sociales" ou encore, que, dans ces temps troublés par les révolutions économiques et politiques, la "pensée dominante est une pensée sociale ${ }^{39}$. On pourrait ne voir là que la redite, un peu trop appuyée certes, de ce que raconte alors son frère sur la nécessaire priorité de l'élévation du prolétariat. Pourtant, bien que se développant dans le cadre vaste et hospitalier de la doctrine du progrès continu, la réflexion économique de Jules Leroux présente plusieurs déplacements intéressants par rapport à la doctrine de Pierre. Lorsque Jules va expliquer que des "questions vitales» ont surgi au présent et doivent être résolues, qu'elles intègrent des doutes et obstacles nouveaux nécessitant une imagination, sociale, nouvelle ce sera pour préciser : «Pourquoi sous le rapport matériel, cette inégalité de richesses parmi les hommes? Pourquoi ceux qui travaillent sont-ils pauvres? Pourquoi cette lutte des machines et des

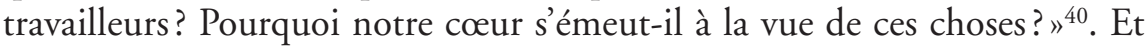
lorsqu'il écrira encore que «le salaire et la pauvreté, en d'autres termes, l'inégalité des hommes, repose principalement sur des phénomènes qui ne sont du ressort que de la science économique», que pour "détruire la pauvreté et le salaire $»^{41}$ on ne peut compter que secondairement sur la morale et la politique, et que des solutions en faveur de l'émancipation doivent être trouvées sur le terrain économique, il semble bien qu'il s'éloigne alors de la doctrine de son aîné qui, lui, faisait de l'émergence de comportements politiques nouveaux dans le cadre du gouvernement représentatif l'alpha et l'oméga de la réforme sociale.

Trois reliefs dominent l'économie politique de Jules Leroux : premièrement, - réflexion sur son outil - il va préciser les rapports complexes, autonomie et complémentarité, que doivent entretenir l'économie politique et la politique au sein de la "science sociale»; deuxièmement, il va faire de l'économie politique une science dont l'objet est la richesse mais en défendant une conception normative de la richesse et en montrant que les finalités

39. Jules Leroux, «Athénée de Marseille. Cours d'économie politique de M. J. Leroux», Le Peuple souverain. Journal du midi, numéro du 4 février 1834. La plus grande partie de ces leçons sera donc reproduite, avec la présentation initiale de Pierre Leroux, dans le volume de la Revue encyclopédique (été 1834), sous le titre «Du salaire». Nous citerons ici à partir des leçons du Peuple sonverain.

40. Jules Leroux, «De l'économie politique considérée comme science», Revue encyclopédique, mars 1833 , p 532-533.

41. Jules Leroux, "Athénée de Marseille», loc. cit., numéro du 5 février 1834. 
de l'activité économique peuvent faire l'objet d'un choix collectif rationnel; troisièmement il va localiser le principal moyen d'accéder à ce but dans la réforme de la propriété mais en montrant comment cette réforme doit procéder en grande partie des actions économiques elles-mêmes.

Face à ces maux sociaux inédits surgissant dans le présent, il faut, explique Jules Leroux, "une science, une science positive, exacte, rigoureuse, mathématique $"$ "2 . L'étude des phénomènes économiques ne date pas d'hier, et des Écoles rivales continuent à s'affronter en ce début des années 1830. Pourtant, le constat dressé par Leroux est sans appel : «la science économique ne se trouve nulle part». Deux tendances s'opposent, en effet, chacune destinée à avorter car niant l'une et l'autre ce qui constitue la propriété même de toute vie, y compris la vie de la connaissance : la relation au milieu. Une première tendance subordonne l'économique au politique ou au religieux. Représentée hier par la Physiocratie, cette tendance aujourd'hui est assumée par l'École saint-simonienne, Leroux évoquant ici «des révélateurs qui subalternisent complètement l'économie politique, et la déduisent comme conséquence de leurs axiomes religieux et sociaux $\|^{43}$; dans cette situation la relation entre éthique et politique d'un côté, économie de l'autre, est trop déséquilibrée pour aboutir à une communication sérieuse. L'autre tendance, représentée hier par Smith, aujourd'hui par des disciples comme Malthus, Ricardo ou Say, isole l'économie de tous ses ancrages sociaux, historiques, politiques et moraux; dans cette seconde tendance, la relation n'existe plus, ou, du moins, n'est plus assumée ouvertement. L'économiste ici, selon les termes de Leroux, "se met en dehors de tout système, de toute théorie sur la société; il isole le fait économique de toute influence étrangère à son point de vue matériel; il le dénature, et recherche, en ce fait ainsi dénaturé, ce qu'il y a tué lui-même, sa loi, sa cause, sa vie». Dans un second temps ce même économiste tente d'insuffler de la vie à son explication : «il fallait après l'avoir complètement isolé, rendre le phénomène économique au milieu qui le fait vivre, et sans lequel il cesse d'être; il fallait expliquer ces étranges influences qu' exercent sur lui les institutions sociales, les phénomènes moraux et intellectuels $»^{44}$. Toutefois, s'étant coupé dès le départ de la vie, sourd à la séquence passé-présentavenir, l'économiste classique est incapable par la suite de la retrouver. Il est dès lors condamné à l'éclectisme et à tous ses dévoiements, Leroux dénonçant au final les conséquences auxquelles aboutit cette économie des Classiques, "conséquences effrayantes par leur athéisme $»^{45}$.

L'erreur commune à ces deux tendances réside dans leur préférence pour "la fixité, l'immobilité» : «tandis que la loi de l'homme est le progrès, tandis que les inspirations secrètes de sa nature respirent hautement encore le pro-

42. Idem, p. 533.

43. Idem, p. 531.

44. Idem, p. 530.

45. Jules Leroux, Le Peuple souverain, loc. cit., numéro du 5 février 1834. 
grès, les faits politiques et économiques sont réputés immuables, la science politique et la science économique ont des formules où le progrès n'a point de place ${ }^{46}$. Endossant ici la doctrine du progrès continu, rappelant sa déclinaison à la condition de l'homme, Jules Leroux montre que ce vitalisme ${ }^{47}$ s'applique aussi éminemment à l'ordre de la connaissance : "Connaitre le fait économique, c'est avoir conscience de sa nature, nature éminemment vivante et progressive, c'est-à-dire persistance en son unité, mobile en sa forme. Bâtir la science économique, sur la connaissance véritable de ce fait, c'est donc lui donner pour base quelque chose de vivant et de progressif, c'est la reconnầtre elle-même progressive et vivante en sa nature $"^{48}$. Pour être à la hauteur de sa tâche, l'économie doit donc progresser, et elle ne peut le faire qu'à trois conditions : définir son individualité, la transformer en communiquant avec la politique et la morale, faire en sorte que cette transformation vérifie et alimente le progrès continu. Revenons sur ces trois points; l'économie se doit de prendre conscience de son individualité. Or, ici, les choses sont apparemment simples : «la science économique repose uniquement sur la connaissance du côté matériel, physique, de nos actes $»^{49}$; mais, selon Leroux, ce "côté matériel» renvoie surtout à des intérêts spécifiques - «l'économie politique, science des intérêts matériels de la société " ${ }^{50}$, écrira-t-il -, intérêts en constante adaptation et qu'il faut donc incessamment observer. L'observation de ces intérêts et des conséquences économiques mais aussi morales ou politiques de leur expression constitue le fondement de l'économie politique : "en s'appuyant directement sur la connaissance entière, exacte, non plus fractionnée ou altérée, du fait, elle peut donc avoir une théorie spéculative aussi exacte, aussi rigoureusement vraie que celle de la science mathématique ${ }^{51}$; il faut également que l'économie politique développe son individualité, progresse, au contact, en relation, avec la politique et la morale; il faut, dans les termes de Leroux, que ces trois disciplines se prêtent un «mutuel appui»; enfin, l'économie politique se développe dans le cadre de la «science sociale». $\mathrm{La}$ «science sociale» devine et traduit le sens général de l'évolution de l'humanité, elle se nourrit des observations partielles des sciences économiques et politiques pour dégager ce sens, et leur offre, en retour, des instructions. Ces instructions vont dans le sens d'une émancipation croissante de l'homme, capable non seulement de s'adapter au milieu, mais aussi de le transformer.

L'homme recherche un "bien-être toujours croissant ${ }^{52}$. Ce bien-être ne désigne pas seulement un stock de biens premiers, mais encore et surtout

46. Jules Leroux, «De l'économie politique», Revue encyclopédique, avril-mai 1833, p. 31.

47. "La vie est partout; elle est dans les êtres, dans les phénomènes, dans les faits, et la vie a le progrès pour loi", ibidem.

48. Idem, p. 34-35.

49. Idem, p. 536.

50. Idem, p. 529.

51. Idem, p. 539.

52. Le Peuple souverain, numéro du 4 mars 1834. 
plus de liberté et plus d'égalité. Cette aspiration n'est pas vérifiée dans le présent, mais, écrit Leroux, on ne peut en accuser ici, n’en déplaise à Malthus, le milieu naturel, une "nature sauvage et rebelle», le véritable coupable étant plutôt «l'état social». L'erreur à ne pas commettre alors, mais dans laquelle est tombée pourtant avec délectation l'économie politique des Classiques, serait de faire de cet état social une nouvelle nature, fixe et impérative. La société, en effet, n'est pas un milieu ou bien elle constitue un milieu unique, singulier : «L'homme n’est point un élément constitutif et passif de la société, être nouveau dans l'échelle des êtres qui vivrait de sa vie propre, écrit Leroux, [...] cette société est, au contraire, une œuvre de l'homme [...], elle a pour but unique celui de toutes ses autres œuvres, de toutes ses actions, d'augmenter son bien-être, de diminuer ses souffrances; en définitive, elle n'est que le milieu enfanté par l'homme pour son plus grand développement " ${ }^{53}$. Si la société est bien l'œuvre de l'homme, résultat de toutes ses actions orientées vers la recherche d'un bien-être croissant, on ne peut alors, dans le présent, "crier et se livrer au désespoir; il faut hardiment mesurer la profondeur de ce problème et se livrer au travail de sa solution ». Ce travail commence dans le domaine de l'économie politique. Science des intérêts matériels, l'économie a concentré ses interrogations sur la définition et les causes de la richesse; "l'économie politique, note Leroux, est la science des richesses " ${ }^{54}$. Anciens et Modernes ont proposé des réponses, et ces réponses constituent le passé de cette science des richesses, charriant leur part d'erreurs, mais aussi de vérités. Leroux mentionnant longuement La Politique d'Aristote, et le Traité d'économie politique de Say souligne que l'erreur commune à ces deux tentatives a été de ne proposer qu'une observation partielle des faits niant le caractère évolutif des institutions et valeurs de leur époque. L'économie d'Aristote traduit et entérine ainsi les intérêts d'une démocratie limitée aux seuls citoyens et légitime l'esclavage, et l'économie de Say, traduction des intérêts d'une aristocratie politique et économique, légitime le salariat et ses inégalités. Mais ces deux tentatives comportent également une part de vérité. L'économie politique des Classiques qui, rappelle Leroux, fut un temps rejointe et transformée par le contexte de 1789, laissait présager une émancipation conquise principalement sur le terrain économique et permettant d'envisager la fin de «l'exploitation de l'homme par l'homme». C'était, aussi, du côté de l'industrie et de la «richesse sociale» que progressaient les valeurs d'égalité et de liberté. Mais, paradoxalement, cet espoir a tourné court quand cette économie politique en est venue à naturaliser cette richesse sociale, faisant de la richesse et de ses sources, non le résultat de jugements et de choix partagés, mais les conséquences d'une seconde nature, sociale, de l'homme. C'est à ce niveau que se constate, selon Leroux, la supériorité des Anciens sur les

53. Le Peuple souverain, numéro du 4 mars 1834.

54. Jules Leroux, «De l'économie politique», loc. cit., p. 48. 
Modernes. L'économie d'Aristote procédant de la distinction entre spéculation naturelle et spéculation artificielle propose une conception normative de la richesse. La richesse est ce qui permet à l'homme, dans la Démocratie athénienne, d'être libre et sage. Loin de traduire un diktat d'un milieu naturel ou social, la richesse est ici la traduction d'un ensemble d'intérêts : «cette définition de la richesse, note Leroux, est bien supérieure à celle des Modernes. Ses conséquences sociales sont la liberté et l'égalité : car elle proclame l'affranchissement définitif de l'humanité des obstacles de tous genres que lui suscitent et les causes qui lui sont extérieures et les causes qu'elle renferme en son sein; car elle donne comme condition, innée dans l'homme, de cet affranchissement, le développement intellectuel le plus grand, le plus grand développement moral et physique " ${ }^{55}$. L'erreur d'Aristote aura été toutefois de lier ce jugement à l'état de la démocratie athénienne, «démocratie étroite et mesquine", n'assurant liberté et égalité qu’à une petite élite de citoyens. Sa conception de la richesse mérite donc d'être désenclavée de cette formule étroite de la démocratie et être sollicitée dans un présent valorisant désormais «le peuple, l'égalité et la liberté, et (...) la démocratie républicaine». Dans ce contexte, une définition générale de la richesse peut alors être proposée : "la richesse est la connaissance des moyens de vivre, et sa source réside dans le triple développement des facultés intellectuelles, morales et physiques de l'homme ${ }^{56}$.

L'économie politique doit donc assumer son statut de science ancillaire permettant le bon gouvernement de la «maison sociale». Le chaos présent s'explique donc, avant tout, par un problème de gouvernement économique. Un obstacle majeur entrave en effet la poursuite de la vraie richesse sociale : «ce qui la gêne, ce qui rend sa réalisation impossible, c'est cette propriété des sources de la vie humaine, de la richesse nationale aveuglément distribuée entre les mains de quelques millions d'hommes; c'est la constitution même de cette propriété, constitution qui a pour résultat nécessaire, fatal, la misère pour le plus grand nombre et l'injustice pour tous ${ }^{57}$. La situation actuelle résulte d'un passé récent, et Leroux signale que la bifurcation s'est opérée, en France, au moment de la Révolution. D'un côté, en effet, la Convention décrète "que l'État doit à chacun de ses membres ce qui est nécessaire à la vie», et, par ce choix inouï, 1793 se donne le moyen de jeter bas les institutions féodales, aussi bien que «les institutions bourgeoises, aristocratiques et commerciales». L'état social nouveau envisagé par la Convention, juge Leroux, «proscrivait complètement l'exploitation de l'homme par l'homme, il détruisait donc le salaire et la bourgeoisie». Pourtant, dans ce contexte révolutionnaire, une autre option s'impose, associant l'émancipation à une libéralisation complète des désirs individuels et à la consécration de ces désirs dans

55. Idem, p. 62

56. Idem, p. 80

57. Jules Leroux, Le Peuple souverain, numéro du 5 février 1834. 
un droit individuel absolu d'accaparement et de possession. C'est ce modèle libéral en doctrine, mais liberticide dans les faits, qui l'a emporté sous l'Empire et la Restauration : «l'ordre social qui prévalut, résume Leroux, fut celui de l'esclavage, de l'injustice et de trois classes ». Dans le même temps, c'est l'économie politique smithienne retravaillée par Say qui théorise ce modèle économique libéral. Say enseigne que les causes de la richesse résident dans la capacité d'une économie spontanément divisée en trois classes à répondre aux fluctuations erratiques de la consommation. L'offre doit s'ajuster strictement et instantanément à la demande et les revenus de chacune des classes ne peuvent jamais longtemps dépasser leur niveau naturel. Pour Jules Leroux, cette économie politique n'est, au mieux, qu'un conte et dissimule les véritables causes ainsi qu'une juste appréhension de la richesse. La proposition suivant laquelle la consommation règle la production n'est en effet juste quà une condition, "qu'à la condition indispensable qu'aucune cause étrangère ne viendra gêner la création de cette production ${ }^{58}$. Or, pour Leroux cette condition n'est pas respectée dans un présent où le véritable régulateur de la richesse est la présence de trois classes inégalement dotées et donc de pouvoir inégal : «les trois classes qui composent cette société sont en réalité les véritables causes régulatrices de la production, et dès lors la production devient à son tour régulatrice de la consommation ${ }^{59}$. La production est clairement insuffisante et cette faiblesse s'explique non par des lois économiques naturelles régissant la production, la consommation et leurs rapports réciproques, mais par sa structure sociale actuelle. Dans l'actuelle société industrielle, la possession des instruments de travail relève de la propriété (le propriétaire), de la location (le maître) ou de l'usage (l'ouvrier). Le propriétaire est oisif et ne fait que consommer (Leroux évoque ici magnifiquement un acteur dont «le désir est le travail ${ }^{60}$ ) ; le maître travaille surtout à transférer les contraintes de la concurrence sur les ouvriers; l'ouvrier, enfin est celui qui utilise les instruments de travail (qu'il a en prêt) pour produire la richesse. Un acteur est donc totalement improductif, le second acteur invente surtout dans le domaine de l'échange ${ }^{61}$ et seul le troisième acteur est véritablement productif. On saisit immédiatement pourquoi cette structure sociale n'est pas efficiente. Mais il faut encore ajouter qu' elle est responsable d'une mauvaise orientation

58. Le Peuple souverain, numéro du 11 mars 1834.

59. Idem, numéro du 11 mars 1834.

60. Jules Leroux, Le Peuple souverain, numéro du 5 mars 1834.

61. La tripartition de Leroux correspond à la situation sociale des années 1830 où l'ouvrier est assimilé à l'artisanat urbain. Son analyse de la fonction des maîtres est assez ambiguë. Il ne cache pas que les maitres peuvent avoir une fonction d'innovation mais ajoute rapidement que, concurrence oblige, leur esprit d'innovation se tourne surtout vers les moyens de transférer les contraintes et chocs sur les ouvriers. Il écrit que le travail du maître consiste à « $1^{\circ}$ diminuer les frais de production, soit en la diminuant réellement [la part du salaire], soit en augmentant la puissance productive de l'instrument de travail, et $2^{\circ}$ augmenter le prix des denrées" (Le Peuple souverain, numéro du 11 mars 1834). Mais lorsque Leroux évoque l'avenir de plus en plus sombre d'un tel régime, acculé à une fuite en avant dans les déséquilibres et les contradictions, il semble abandonner la tripartition pour ne plus évoquer qu'une lutte frontale entre propriétaires et ouvriers (ces derniers ayant absorbés l'ancienne classe des maîtres). 
de la production. Leroux rappelle que «les propriétaires vivent de la production; les maîtres et les ouvriers, par la production" et il dira, formule plus paradoxale, que «les ouvriers et les pauvres sont les parasites des riches ${ }^{62}$. En fait, dans le système économique présent, la minorité de propriétaires oriente tant quantitativement que qualitativement la production, et ses moindres desiderata déclenchent, auprès de la majorité des maitres et des ouvriers, une lutte de tous les instants. Un tel système, ajoute Jules Leroux ne peut persister qu'en accentuant constamment ses contradictions et ses déséquilibres : il lui faudrait donc, pour perdurer, encourager, d'un côté, «l'immoralité des excès» et, d'un autre côté, «l'immoralité de l'abnégation » ${ }^{63}$.

Mais cette évolution est impossible tant elle est contraire à la doctrine du progrès continu et à l'aspiration des hommes pour l'égalité et la liberté. Ce n'est pas une recherche sur les causes de la richesse des nations qu'a menée Say, explique Leroux, mais bel et bien son contraire : cette économie politique, observe-t-il, enseigne le gaspillage, le désordre et la sous-production, et cette économie est peuplée d'aliénés et de mendiants. Une autre recherche s'impose donc: "connaissant la nature trinaire de la société actuelle et la nature des causes constitutives de ces trois classes, trouver en ces natures les moyens d'arriver à un état de société où régneront tout à la fois l'égalité, la liberté et l'unité». Il faut, au présent, transformer l'économie pour accéder à un niveau satisfaisant de richesse : un montant total de biens, mais aussi une organisation permettant l'affirmation d'hommes libres, sages et égaux. Se distançant de son frère Pierre, Jules Leroux montre alors que cette transformation doit prioritairement s'opérer dans le domaine économique. Naturellement, l'avènement progressif d'un gouvernement républicain, pleinement représentatif, peut accompagner et renforcer cette transformation économique. Mais la solution procède surtout d'une modification de l'organisation économique elle-même : il faut "changer la constitution de la propriété»" explique-t-il. Mais dépassant les simples allusions faites sur ce thème par son aîné Pierre, Jules Leroux précise très clairement ce qu'il entend par là : la possibilité d'associer encore plus concrètement les ouvriers en expérimentant une coopérative de production ${ }^{65}$.

Ce n'est pas dans les pages de la Revue encyclopédique que Jules Leroux s'explique précisément sur ce changement mais dans son adresse Aux ouvriers typographes. Publiée en 1833 sa brochure porte en sous titre, De la nécessité de fonder une association ayant pour but de rendre les ouvriers propriétaires des instruments de travail. Le début des années 1830 marque un changement majeur de régime typographique, les techniques d'impression, l'édition et la

62. Jules Leroux, Le Peuple souverain, numéro du 12 mars 1834.

63. Idem, numéro du 12 mars 1834.

64. Idem, numéro du 12 mars 1834 .

65. Une perspective qui le rapproche ici des propositions faites peu avant par Philippe Buchez dans son journal L'Européen, journal des sciences morales et économiques en 1831. 
librairie évoluant dès lors très rapidement. On se situe dans un contexte de doutes pour les ouvriers typographes qui affrontent une crise qui les dépasse, la misère touchant "la classe entière ${ }^{66}$. Dans cette situation, Jules Leroux engage les ouvriers à ne pas verser dans l'égoïsme : la rhétorique libérale enseigne que "la classe n'existe pas; il n'y a que des individus", mais il ne faut pas ici être dupe, car cet individualisme, «n'est pas la liberté, c'est l'isolement, l'isolement complet ${ }^{67}$. Il ne faut pas croire ceux qui lient le malheur actuel à des phénomènes économiques objectifs, les retournements naturels de la conjoncture (les crises que l'on commence à suspecter périodiques) ou encore l'innovation technologique (les machines). Il y a réellement une crise, mais face à cet obstacle il faut agir, et agir collectivement pour s'adapter à cette situation, la transformer; il faut viser, selon les termes de Jules Leroux, un «but d'organisation, d'organisation intérieure». En bref, explique-t-il, il faut créer une "association typographique " ${ }^{68}$. Dans un premier temps, cette association doit viser à réformer connaissances et habitudes : il faut une "enquête véritable» sur les tarifs, sur l'introduction des machines, sur l'organisation du travail, et il faut «que la division libre, mais réglée, soit ouverte sur l'enquête » ${ }^{69}$. Cette enquête est un préalable à l'action, elle révélera que, là comme ailleurs, le problème pseudo-objectif est en fait un problème social, donc négociable, elle révélera que «la question est une question de propriété, d'affranchissement». Selon Leroux, «un vaste atelier $»^{70}$, comme l'expérimentent actuellement les tailleurs, mériterait d'être créé. Il permettrait d'apprendre à réguler nombre de problèmes de cette industrie : régularisation des commandes, de l'apprentissage et de l'accès à la profession, des salaires, de l'assistance aux ouvriers sans emploi, aux malades, etc. Au final cette tentative de coopération ne serait d'ailleurs pas si anodine et ponctuelle tant sur le plan économique que politique, Jules s'exclamant finalement : «Aujourd'hui, nous pourrions créer sur le sol de la France, au sein de la capitale, une imprimerie que nous exploiterions nous-mêmes; nous pourrions entrer dans la voie de la concurrence avec nos maitres, ou plutôt, devant notre imprimerie colossale, toutes les leurs s'écrouleraient ${ }^{71}$.

Développée entre 1831 et 1835 dans les pages de la Revue encyclopédique par Pierre Leroux, la doctrine du progrès continu abrite de nombreuses idées extrêmement fécondes en économie politique. La critique du rationa-

66. Jules Leroux, Aux ouvriers typographes. De la nécessité de fonder une association ayant pour but de rendre les ouvriers propriétaires des instruments de travail, Paris, imprimerie L. E. Herhan, 1833, p. 4.

67. Idem, p. 10.

68. Idem, p. 5.

69. Idem, p. 5.

70. Idem, p. 13

71. Idem, p. 15 
lisme classique et de son avatar économique est fondamentale. De même, Leroux ne privilégie pas un évolutionnisme aveugle mais, considère, selon une orientation normative forte, que l'économie politique doit réfléchir et a les moyens de réfléchir non seulement sur les moyens, mais aussi sur les buts de l'activité économique. Cette option permet à ce projet de déboucher sur une conception gradualiste de la réforme qui place les enjeux économiques et sociaux au premier plan. L'approche de Leroux n'est toutefois pas sans lacunes. Il semble bien que pour lui, la conquête du pouvoir politique, et l'exercice démocratique de ce pouvoir épuise la réforme sociale. Sa doctrine du progrès continu et de la perfectibilité pivote autour de la notion de communication - "c'est cette faculté de communiquer qui est la base et l'essence de la société» écrit-il en $1835^{72}$ - et on pourrait appliquer à cette doctrine certaines critiques adressées aujourd'hui à la notion habermassienne « d'espace public $»^{73}$. Si la réflexion de Pierre Leroux mobilise en priorité une approche en termes de communication, celle, plus localisée, de son jeune frère paraît plus volontiers pencher vers une approche en termes d'investigation ${ }^{74}$. Cette option conduisit chez Jules à deux types de résultats. En premier lieu, les thèses économiques de Pierre, sur la rationalité, l'évolution, la possibilité de choix et actions communs négociés, sur les priorités et voies de la réforme, furent nettement approfondies; en second lieu, Jules Leroux aperçu bien plus nettement que son aîné, les complémentarités entre économie politique et politique et il montra comment les transactions et autres expériences économiques elles-mêmes pouvaient et devaient participer de façon décisive aux progrès de l'émancipation.

Ludovic Frobert est directeur de recherche au CNRS, TRIANGLE/ENS-LSH Lyon

72. Pierre Leroux, «Des rapports du christianisme avec la doctrine philosophique du progrès», loc. cit., p. 310 , note 1 .

73. Nancy Fraser, 'Rethinking the public sphere: A contribution to the critique of actually existing democracy'; Geoff Eley, 'Nations, Publics, and Political Cultures: Placing Habermas in the NineteenthCentury', in Craig Calhoun (ed), Habermas and the Public Sphere, Cambridge, MIT Press, 1993, respectivement p. 109-142 et p. 289-339.

74. Sur la distinction entre communication et investigation, et sur l'origine de cette notion d'investigation chez John Dewey, voir ici Hilary Putnam, Définitions, traduit de l'anglais et présenté par C. Bouchindhomme, Combas, Éditions de l'Éclat, 1992. 